\title{
GIUSEPPE TARTINI E LA PROPRIETÀ INTELLETTUALE DELLA MUSICA NEL SETTECENTO
}

\author{
GUIDO VIVERIT \\ Università degli Studi di Padova
}

\begin{abstract}
Izvleček: Vrazpravi so obravnavani različni vidiki Abstract: This paper examines various aspects pojma intelektualne lastnine glasbe, kot ga je pertaining to the concept of intellectual property of razvil Giuseppe Tartini. Ta pa se ne nanaša zgolj music developed by Giuseppe Tartini, concerning na njegove lastne skladbe, temveč tudi na dela not only his own compositions, but also the works drugih glasbenikov (vključno z njegovimi učenci). of other musicians (including his own students).

Ključne besede: Giuseppe Tartini, intelektualna Keywords: Giuseppe Tartini, intellectual properlastnina, Pasqualino Bini, glasbeno založništvo, ty, Pasqualino Bini, Music publishing, Antonio Antonio Quartieri, Giovanni Battista Martini. Quartieri, Giovanni Battista Martini.
\end{abstract}

La circolazione della musica nell'Europa del Settecento, sia in forma manoscritta sia a stampa, avvenne perlopiù senza l'autorizzazione e senza il controllo di quelli che oggi definiremmo i legittimi proprietari dell'opera, fossero essi autori, editori o committenti. ${ }^{1}$ Le ragioni di tale fenomeno si devono ricercare nel progressivo emergere di nuovi mercati (soprattutto in Gran Bretagna e Francia) e nell'assenza o nella scarsa efficacia di strumenti di tutela della proprietà intellettuale, che furono definiti solo a partire dalla fine del sec. XVIII, assumendo le forme del diritto d'autore, nell'Europa continentale, e del copyright, in area anglosassone.

Gli strumenti normativi con i quali, nel corso del Settecento, si tentò di porre un freno alla diffusione non autorizzata della musica di un autore o alla pubblicazione di ristampe 'pirata' da parte di un editore, furono differenti da paese a paese. ${ }^{2}$

La principale forma di protezione esistente in buona parte dell'Europa continentale e in Gran Bretagna fu il privilegio di stampa. ${ }^{3}$ Concesso dalle autorità del paese in cui veniva richiesto, esso garantiva all'editore la possibilità di pubblicare un'opera, per un limitato periodo di tempo ed entro determinati confini territoriali, proibendo agli editori privi di tale privilegio di stampare le opere 'protette'. Nel caso in cui il possessore del privilegio

1 Sull'argomento si vedano Rasch, Music Publishing; Lenneberg, The Dissemination of Music.

2 Cfr. Guillo, "Legal Aspects," 115-138.

3 Ibid., 122; De Vecchis et al., La proprietà del pensiero, 13-16. 
fosse stato l'autore, costui aveva la facoltà di affidare la pubblicazione della propria opera allo stampatore che avesse ritenuto più idoneo. Le modalità di concessione e i tempi di durata del privilegio, così come le sanzioni per i trasgressori, erano differenti a seconda delle norme stabilite dalle autorità che concedevano il privilegio stesso.

Nel caso della Gran Bretagna, un altro strumento utilizzato fu il Copyright Act, promulgato nel 1710 dalla regina Anna. ${ }^{4}$ Esso stabiliva il diritto, per l'autore, "di far stampare a suo piacimento la propria opera per un determinato numero di anni, ricavandone profitto." ${ }^{5}$ Nella maggior parte dei casi, tuttavia, era l'editore ad assicurarsi il copyright per l'opera, acquistandone il diritto dall'autore. Il Copyright Act tutelava principalmente i testi letterari. Solo nel 1777 il King's Bench (la Corte del Banco del Re) si pronunciò in merito alle composizioni musicali. In occasione di una causa che vide contrapposti Johann Christian Bach e gli editori Longman, Lukey \& Co., per alcune musiche pubblicate da quest'ultimi senza il consenso dell'autore, il King's Bench stabilì che anche le composizioni musicali dovessero essere considerate soggette alla protezione prevista dal Copyright Act. ${ }^{6}$

Le forme di tutela considerate difendevano la proprietà dell'edizione, non quella dell'opera, favorendo perciò l'attività dell'editore, piuttosto che quella dell'autore, per il quale l'unico vantaggio derivava dalla vendita del manoscritto della composizione e dagli accordi raggiunti con lo stampatore per la prima edizione.

In un simile contesto si trovò ad operare Giuseppe Tartini. Attraverso alcuni documenti, in parte inediti, questo articolo intende gettare luce sull'idea di 'proprietà intellettuale della musica' sviluppata da Tartini, intendendo con questo concetto, nell'ambito considerato, l'insieme di aspetti che riguardano:

- il riconoscimento della paternità dell'opera;

- il livello di controllo dell'autore sulla stessa;

- il rispetto della volontà dell'autore;

- il riconoscimento di un adeguato valore economico dell'opera.

Questo breve saggio si articola in tre sezioni. Nella prima sarà trattato il concetto di proprietà intellettuale sviluppato da Tartini in rapporto alle proprie composizioni; la seconda e la terza parte saranno dedicate alla presentazione di un esempio relativo al comportamento adottato da Tartini nel caso di musiche composte da un suo allievo e da un autore estraneo alla sua cerchia. ${ }^{7}$

La musica di Tartini si diffuse ampiamente sia in forma manoscritta sia a stampa. ${ }^{8}$ In

4 Si vedano in particolare Hunter, "Music Copyright in Britain," e Small, "The Development of Musical Copyright." Sono grato al prof. Michael Talbot per avermi gentilmente segnalato quest'ultimo contributo.

5 De Vecchis et al., La proprietà del pensiero, 29.

6 Small, "Johann Christian Bach," e Id., "The Development of Musical Copyright," 329-354, 360-370. Di rilevante interesse anche i casi trattati in Rabin et al., "Arne, Handel, Walsh."

7 Sul diritto d'autore e sulla percezione che di esso avevano sviluppato i compositori vissuti tra il XV e il XVIII secolo si veda Pohlmann, Die Frühgeschichte.

8 Sulla tradizione manoscritta, in particolare dei concerti, si veda Viverit, "Dissemination and Tradition." Sulle edizioni di musica tartiniana si vedano: Fanzago, Orazione del signor abate, 45; Brainard, "Le Sonate a tre," 104-05; Brainard, Le sonate per violino, xxxv-xl; Bisi, "Contributo 
quest'ultimo caso solo le opere I e II furono autorizzate senza dubbio dall'autore; tutte le altre pubblicazioni furono, con molta probabilità, realizzate senza il suo consenso o a sua insaputa. ${ }^{9}$ Alcuni documenti relativi alle stampe autorizzate costituiscono una fonte di informazioni imprescindibile per conoscere le condizioni che Tartini propose agli editori per pubblicare le sue composizioni. ${ }^{10}$

Le opere I e II, entrambe raccolte di dodici sonate ciascuna, furono stampate a distanza di undici anni l'una dall'altra. La prima raccolta uscì nel 1734, ad Amsterdam, per i tipi dell'editore olandese Michel Charles Le Cène, uno dei più celebri editori musicali europei del primo Settecento. ${ }^{11} \mathrm{Nel} 1745$, a Roma, Antonio Cleton, incisore, decisamente meno noto, attivo tra il 1730 e il 1769, pubblicò l'Op. II. ${ }^{12}$

Le condizioni che il compositore concordò con entrambi gli editori per la stampa delle proprie musiche sono citate, piuttosto dettagliatamente, in un passo della lettera del 7 gennaio 1745, inviata da Tartini al suo corrispondente di Rovereto, Giuseppe Valeriano Vannetti:

Con Monsieur Le Cene senza replica alcuna di lettere si è fatto e concluso di mia proposizione l'accordo di zecchini 72 veneziani per le dodici mie sonate già stampate a violino solo, di esemplari 50 per me e della dedica a mio utile e conto; e, consegnate le suonate mie manoscritte in Venezia al Pomer, nell'atto stesso furono pagati li zecchini 72. Lo stesso identico accordo ho fatto presentemente in Roma $\left[\ldots . .{ }^{13}\right.$

L'accordo tra compositore ed editore prevedeva perciò:

- il pagamento immediato al momento della consegna delle composizioni di 72 zecchini veneziani;

- la consegna di 50 esemplari per uso privato;

- la possibilità di introdurre l'opera con una lettera dedicatoria, richiesta, quest'ultima, che comportava per l'autore maggiore prestigio e un ulteriore vantaggio economico. ${ }^{14}$

Le principali ragioni che indussero Tartini a pubblicare le due edizioni di sonate appaiono di diversa natura. L'Op. I fu pubblicata, probabilmente, con l'intenzione di evitare

ad un'edizione critica;" Marconato, "Per un'edizione critica," 14-17; Ruggeri, "Contributo per un'edizione critica," 11-30; Durante, "Tartini and his Texts;" Canale Degrassi, "I concerti solistici di Giuseppe Tartini," 54-62.

9 Tra le raccolte qui considerate sono escluse le rielaborazioni di composizioni di Tartini pubblicate nel corso della sua vita.

${ }^{10}$ Sul rapporto tra Tartini e i suoi editori si veda Durante, "Tartini and his Texts," 180-185. A questo riguardo si vedano anche gli accordi che coinvolsero alcuni compositori contemporanei di Tartini in Rasch, "«Il cielo batavo»."

${ }^{11}$ Sonate a Violino e violoncello o cimbalo. Dedicate a Sua Eccellenza il Signor Girolamo Ascanio Giustiniani da Giuseppe Tartini. Opera prima. Amsterdam. Spesa di Michele Carlo Le Cene [Numero editoriale: 576]. Cfr. Brainard, Le sonate per violino, xxxv-xxxvi.

${ }^{12}$ Sonate a violino e basso dedicate al S. ${ }^{r}$ Guglielmo Fegeri di Giuseppe Tartini. Opera seconda. Antonius Cleton Scul: Romae Superiorum permissu. Cfr. Brainard, Le sonate per violino, xxxvi. Su Antonio Cleton si veda Rostirolla, "L'editoria musicale a Roma," 153-154.

${ }^{13}$ Pasini, "Il Tartini a Giuseppe Valeriano Vannetti," 6-8.

${ }^{14}$ Per l'Op. I Tartini ricevette da Girolamo Ascanio Giustinian, suo allievo e dedicatario della raccolta, 40 zecchini in segno di riconoscimento. Cfr. Vio, "Note biografiche," 63. 
la circolazione di musiche non autorizzate: così fa intendere un passo della lettera del 31 marzo 1731 che Tartini inviò a padre Giovanni Battista Martini: "[Non le ho risposto perché] sinora sono stato e son attualmente occupato nello scrivere per mettere in stampa dodeci sonate a solo, non per mia volontà, ma forzato da una cattiva azione fattami da un stampatore olandese." ${ }^{\prime 15}$ Visto il buon esito dell'Op. I, Tartini comprese che la stampa delle sue musiche poteva rappresentare un guadagno significativo, perciò le ragioni che lo indussero all'edizione dell'Op. II furono probabilmente di natura economica. A supportare questa ipotesi è soprattutto una lettera che Tartini scrisse, il 14 febbraio 1746, al fratello Pietro: "Ho presentemente un capitale di 1500 ducati arenato in un cassone dietro la porta di casa mia in tante stampe che non possono esitarsi né addirizzarsi in altri luoghi a cagione delle guerre presenti. E sopra più ho per le medesime 200 duc(ati) di debito. Ho creduto di raddoppiare il capitale, lo perderò almeno mezzo."16

Da questa lettera apprendiamo che Tartini:

\begin{abstract}
- custodiva nella propria casa tante stampe dell'edizione di sonate per un valore di 1500 ducati; ${ }^{17}$

- si occupava in prima persona della spedizione delle stampe non solo entro i confini della Repubblica di Venezia, ma anche verso i paesi coinvolti dalle "guerre presenti". Con quest'ultima espressione Tartini si riferiva con tutta probabilità alla guerra di successione austriaca, che si combatté, tra il 1740 e il 1748, in Boemia, Germania, nelle Fiandre e nell'Italia settentrionale;

- per le stampe aveva contratto un debito di 200 ducati e, se inizialmente pensò di raddoppiare il capitale, si rese conto successivamente che ne avrebbe perso almeno metà a causa delle difficoltà contingenti. Tuttavia, le parole di Tartini non permettono di precisare a quanto ammontasse l'intero investimento iniziale. ${ }^{18}$
\end{abstract}

Si può ipotizzare, infine, che Tartini considerasse una terza importante ragione per la stampa delle proprie composizioni la necessità di farsi conoscere al di fuori d'Italia, per alimentare la fama dalla quale dipendeva anche la sua attività di insegnante. ${ }^{19}$

Tuttavia, come già accennato, la circolazione delle musiche del piranese, manoscritte e a stampa, avvenne perlopiù in forme non autorizzate. Ciò dipese dalla destinazione delle fonti musicali e dalle modalità di trasmissione delle stesse. I manoscritti di concerti e sonate di Tartini servirono in parte per la sua attività concertistica presso la cappella musicale della basilica del Santo di Padova o per esecuzioni in accademie, in parte per

${ }^{15}$ Cfr. Durante, "Tartini and his Texts," 181-182.

${ }^{16}$ Hortis, "Lettere di Giuseppe Tartini," 220. Hortis identifica in Domenico, un altro fratello di Giuseppe Tartini, il destinatario della lettera. L'originale della lettera si trova presso l'Archivio Regionale di Capodistria - Sezione di Pirano; SI PAK PI 334, busta 2, n. 48. Ringrazio il dottor Alberto Pucer per queste informazioni. Si vedano anche Brainard, "Die Violinsonaten," 42-45 e Durante, "Tartini and his Texts," 180.

${ }^{17}$ Secondo Brainard le copie in possesso di Tartini dovevano essere oltre 385. Si veda Brainard, "Die Violinsonaten," 44.

${ }^{18}$ Tartini quantificò i guadagni totali derivanti dalla vendita di stampe e composizioni manoscritte rispettivamente in 300 e 200 ducati. Si vedano in proposito alcuni documenti tartiniani trascritti in Frasson, "Giuseppe Tartini," 148-152.

${ }^{19}$ Sulla ‘Scuola delle Nazioni' si vedano Petrobelli, "La scuola di Tartini;," Canale, "Fonti per una ricostruzione," e Viverit, "Dissemination and Tradition." 
la fornitura di musica ad uso di committenti privati, e, probabilmente, anche come strumento per l'attività didattica. Dai manoscritti dei concerti giunti sino a noi è possibile ipotizzare che Tartini, come del resto avveniva per tutti i compositori di un certo rilievo, abbia redatto le sue composizioni quasi esclusivamente in forma di partitura. Il compito di copiare le singole parti dei concerti per le esecuzioni o per altre destinazioni, avvenne ad opera di copisti di professione, in qualche caso di suoi allievi o di musicisti dilettanti. Perciò si può ipotizzare che un numero consistente di persone abbia avuto accesso alle sue musiche, seppure, presumibilmente, in condizioni di controllo da parte dell'autore. Quindi la proprietà, il controllo e la diffusione delle sue composizioni furono strettamente connessi al livello di fedeltà, correttezza e rispetto dei suoi più stretti collaboratori.

Quando gli strumenti di tutela della propria musica, si rivelarono inefficaci, la reazione di Tartini dovette assumere la forma di dura condanna morale nei confronti di chi aveva 'trafugato' le sue composizioni. Ne è un esempio la lettera che Tartini inviò, il 12 marzo 1750, ad uno dei suoi più noti corrispondenti, il conte Francesco Algarotti, che allora ricopriva l'incarico di ciambellano di Federico II re di Prussia. ${ }^{20}$ A proposito di sei concerti inviati al principe Ferdinand Philipp von Lobkowitz, Tartini scrisse:

La mia delicatezza mi obbliga parimenti fargli sapere, che ho occasione di dubitare, che il copista mi abbia trafugato con doppia copia uno di questi sei concerti; et è quello in b mi, terza minore. Non son sicuro, ma molto temo. Mi son espresso abbastanza col medesimo; ma se lo ha trafugato, nulla gioverà, perché l'animo è vile, et il bisogno è a proportione della miseria. Altrettanto ciò mi dispiace, quantoché è un concerto di un genio particolare, sopra cui avrò piacere di rilevare il giudizio e sentimento di Sua Altezza; e prescindendo da un solo passo a doppia corda, Sua Altezza lo può eseguire con facilità. ${ }^{21}$

Questi documenti testimoniano l'interesse di Tartini rispetto alla proprietà della musica da lui stesso composta, nonché il tentativo di gestirne la diffusione attraverso il controllo dell'attività dei suoi copisti e con proposte di accordi con gli editori: iniziative che si rivelarono spesso senza successo. Tuttavia, se è innegabile che la consistente circolazione non autorizzata delle sue musiche rappresentò per lui un danno economico, non c'è dubbio che essa contribuì in maniera rilevante a consolidare la sua fama europea. ${ }^{22}$

Di particolare interesse risulta anche l'atteggiamento che Tartini adottò in rapporto alla musica di proprietà di uno dei suoi numerosi allievi. Alcune lettere oggi conservate presso il Museo internazionale e biblioteca della musica di Bologna, appartenenti al carteggio di Giovanni Battista Martini, datate tra la fine del 1739 e l'inizio del 1740, gettano luce sulla trasmissione di alcune partiture composte da uno degli allievi prediletti di Tartini e

${ }^{20}$ Sul rapporto tra Tartini e Algarotti si veda Petrobelli, “Tartini, Algarotti," e Id., "La scuola di Tartini," 81-82.

${ }^{21}$ Il testo della lettera è tratto da Lozzi, "Tartini e Bini," 183. La composizione trafugata è identificabile col concerto per violino e archi in Si minore, D 125. Si veda in proposito Durante, "Tartini and his Texts," 178, nota 23. Su Ferdinand Philipp Joseph von Lobkowitz (1724-1784), dedicatario dei concerti, si vedano Brainard, "Die Violinsonaten," 94-95; Petrobelli, "Una presenza di Tartini," 65, nota 2 e Petrobelli, "La scuola di Tartini," 81-82.

22 Viverit, "Dissemination and Tradition." 
inviate al piranese attraverso una serie di intermediari, seguendo un percorso che prende il via da Roma per concludersi a Padova.

Il 3 dicembre 1739 Tartini scrisse a Martini di aver ricevuto alcune partiture poste dentro una cappelliera e lo ringraziò per avergliele fatte recapitare a Padova per mezzo del Padre Maestro Giovanni Paolo Cesarotti. ${ }^{23}$ Dopo i ringraziamenti, aggiunse: "[...] si degni di scrivermi dà chi hà ricevuto le suddette partiture e se erano sciolte, come le hò trovate io nella Cappelliera. La suplico di questa informazione come necessaria per evitar qualche disordine, che potrebbe seguire, se io non sapessi il tutto." ${ }^{24}$

All'incirca dopo tre mesi, il 26 febbraio 1740, non avendo, con tutta probabilità, ricevuto risposte soddisfacenti, Tartini scrisse a Padre Martini una nuova lettera, che ci offre una ricostruzione piuttosto dettagliata dell'itinerario che seguirono le partiture, con un elenco di tutti coloro che ne entrarono temporaneamente in possesso e con un accenno al proprietario delle stesse.

Bisogna [...] che $\mathrm{V}$ (ostra) R(iverenza) abbia la bontà di sopportarmi in ciò, che sono per dirle di nuovo circa le consapute partiture del mio Scolare di Roma, che capitorno in Sue mani. Le medesime furono mandate sigillate con quattro sigilli al Sig(no)r Antonio Quartieri, et egli tali e quali le consegnò al Sig(no)r d(o)n Francesco Benzoni di lei amico, acciò le dasse al $\mathrm{P}(\mathrm{ad}) \mathrm{re} \mathrm{M}$ (aestr)o Baldazzi, che veniva costì, come fece pontualm(en)te nello stesso giorno. Il $\mathrm{P}(\mathrm{ad}) \mathrm{re} \mathrm{M}$ (aestr)o Baldazzi, le avrà portate $\mathrm{a} \mathrm{V}(\mathrm{ostra}) \mathrm{R}$ (iverenza) in Bologna; egli non è dilettante di musica, onde naturalm(en)te dovrebbe averle consegnate a V(ostra) R(iverenza) tali quali sono state consegnate a lui. Mi faccia la carità senza niun riguardo dirmi com'è, o come può essere questo fatto, perché in sostanza sappia, che a me null'altro importa, se non che quella robba non sia stata copiata, che sia stata veduta, non m'importa nulla; sono partiture del mio scolare, che mi mandava con gelosia, e mi dispiacerebbe, che si pubblicassero, perché lo Scolare potrebbe sospettare, che questo male lo avessi fatto io. Purché ciò non possa esser seguito, del resto le replico, che non m'importa un quattrino. Onde per mia intiera quiete, e senza un'imaginabile riguardo mi dica schiettam(en)te, se devo credere al Quartieri, con cui mi sono lamentato fortem(en) te. Mà egli ([?] santo uomo onesto come sà V(ostra) R(iverenza)) mi hà scritto sinora trè lettere, protestando, e arciprotestando con testimoni, che il fagottino, consegnato al $\mathrm{P}(\mathrm{ad}) \mathrm{re} \mathrm{M}$ (aest)ro era sigillato con quattro sigilli. Abbia pazienza di risponder anco per questa volta sopra tal fatto $[\ldots]^{25}$

Tra le numerose lettere che formano il carteggio martiniano è stato possibile individuarne una, che aggiunge alla vicenda alcuni tasselli mancanti. Si tratta di una lettera che Francesco Benzoni, uno degli intermediari citati da Tartini, inviò da Rimini, il 14 novembre 1739, a padre Martini:

Per mano del $\mathrm{P}(\mathrm{ad})$ re M(aest)ro Baldazzi che viene a predicare a Coreggio [...] li sarà consegnato [...] un involto a lei diretto sigillato, ma questo è venuto da Roma, che l'ha

${ }^{23}$ Si tratta quasi certamente del minore conventuale Giampaolo Cesarotti, Padre Guardiano del Convento del Santo a Padova nel 1736. Si veda Sartori, Archivio Sartori, 642. Ringrazio il Direttore della Pontificia Biblioteca Antoniana di Padova, padre Alberto Fanton, per avermi segnalato questa informazione.

${ }^{24}$ Museo internazionale e biblioteca della musica di Bologna (I-Bc), Carteggio Martini, I.017.015.

${ }^{25}$ I-Bc, Carteggio Martini, I.017.017. 
mandato il Sig(no)r Pasqualino Bini scolaro del Sig(no)r Tartini ad effetto si mandi a lei, e che lei poi lo faccia capitare a Padova in mano del Sig(no)r Don Antonio Vandini. Quest'è l'incomodo che ci devo reccare per parte del Sig(no)r Bini che distintam(en)te la riverisce, siccome fà il Sig(no)r Quartieri che sempre suo se le prottesta. [... $]^{26}$

Apprendiamo da quest'ultima lettera che l'involto doveva essere consegnato al violoncellista Antonio Vandini, amico e collega di Tartini presso la cappella musicale della basilica del Santo, che frequentemente faceva la spola tra Padova e Bologna, portando con sé lettere e materiale da consegnare a padre Martini o a Tartini. ${ }^{27}$ Per ragioni che non conosciamo le partiture e la lettera furono affidate al Padre Maestro Cesarotti.

Le partiture appartenevano a Pasqualino Bini (1716-1770), allievo di Tartini all'incirca tra il 1731 e il 1734 e attivo a Roma come violinista virtuoso. ${ }^{28}$ Nessuna composizione di Bini fu pubblicata, e non è possibile stabilire al momento relazioni tra le partiture indicate nella lettera e i manoscritti di sue composizioni oggi esistenti. Non conosciamo la risposta di Martini e non possiamo sapere chi effettivamente ruppe i sigilli. Ciò che qui interessa rilevare riguarda la prassi di trasmissione della musica e il comportamento di Tartini. Nel tragitto da Roma a Padova le partiture passarono per le mani di sei persone. Tartini dimostrò un'estrema responsabilità, agendo per la segretezza delle composizioni del suo allievo. Scrisse di essersi "lamentato fortemente" con Antonio Quartieri, un violoncellista virtuoso di Rimini che Tartini conosceva bene, unico musicista oltre a Martini potenzialmente interessato a voler copiare le composizioni. ${ }^{29} \mathrm{E}$ affermò, Tartini, di aver ricevuto dal Quartieri tre lettere in cui il riminese si difese dall'accusa, addirittura coinvolgendo testimoni che confermassero la sua versione. La principale preoccupazione di Tartini era che la musica non fosse stata copiata, perché "dispiacerebbe che si pubblicassero, perché lo Scolare potrebbe sospettare che questo male lo avessi fatto io."

Tartini si dimostrò altrettanto scrupoloso nei confronti di alcune musiche composte da un altro musicista, questa volta estraneo alla sua cerchia. Il 23 febbraio 1766, Tartini scrisse una lettera ad un "suo Padrone dilettante di Musica in Venezia". Il contenuto della missiva ci è noto grazie alla trascrizione che ne fece l'abate Francesco Fanzago in occasione dell'orazione funebre in onore del piranese:

Ho ricevuta, e consegnata la seconda parte dell'opera quinta del Corelli ridotta in conserti dal Geminiani, al copista da me già soddisfatto per la copia della prima. Circa la variazione che non le piace, e vuol cambiata, V(ostra) S(ignoria) Illustriss(ima) mi perdoni, in hoc non laudo. Né ella, né io, né quanti siamo, possiamo ragionevolmente arrogarsi questa libertà. Si può per forza, ma ingiuriando il compositore; troppe sono

${ }^{26}$ I-Bc, Carteggio Martini, I.023.083.

${ }^{27}$ Su Antonio Vandini si veda Hansell e Massaro, "Vandini, Antonio," e Barazzoni, "Vandini, Antonio." Testimonianza dello stretto rapporto che intercorse tra Vandini e Martini sono le numerose lettere conservate nel Carteggio Martini in I-Bc.

${ }^{28}$ Su Pasqualino Bini e le sue composizioni si vedano Radiciotti, "Aggiunte e correzioni," 582-586; Dizionario Enciclopedico della Musica e dei Musicisti, s.v., "Bini, Pasquale (Pasqualino);” Duckles et al., Thematic Catalog, 61-65.

${ }^{29}$ Antonio Quartieri fu violoncellista e maestro di cappella presso la Cattedrale di Rimini. Vedi Ferretti, "Giuseppe Tartini e le Marche," 49. 
le cose musicali che non incontrano i genj particolari. Ella deve accordarmi che non per questo chi non le gradisce ha autorità di cambiarle: ha bensì autorità di non volerle per proprio uso. Ma che a Lei accomodi tutta l'opera: non accomodi quella variazione, e però la voglia cambiata a fronte di tutta l'opera ottima, e approvata, durus est sermo hic, almeno alle mie orecchie. Da buon servitore le dico il mio sentimento, e poi Ella faccia pure quello le par, e piace. Ma su questo punto mi rescriva, e decida, perché il copista da me ha ricevuto l'ordine di non proseguire la copia quando sia arrivato a quel segno, se prima non è da me avvisato di ciò che deve fare. $[\ldots]^{30}$

Sebbene non sia oggi reperibile la fonte autografa, non ci sono elementi che inducano a considerare il testo non autentico. Al momento non è possibile nemmeno identificare il destinatario della lettera: per chiarire questo aspetto, potrebbe rivelarsi utile un'indagine approfondita della copia dell'Op. V di Corelli "ridotta in Concerti da Francesco Geminiani", conservata in uno dei fondi tartiniani più importanti: la Jean Gray Hargrove Music Library dell'Università della California a Berkeley. ${ }^{31}$

Dal testo della lettera appare ancora più chiara la posizione di Tartini riguardo ai principali aspetti relativi alla proprietà intellettuale. Alla richiesta di apportare modifiche ad una variazione dei concerti, Tartini rispose con fermezza: "Né ella, né io, né quanti siamo, possiamo ragionevolmente arrogarsi questa libertà. Si può per forza, ma ingiuriando il Compositore." L'unica autorità che ha chi non gradisce le variazioni originali, scrisse Tartini, è di "non volerle per proprio uso". Una scelta di espressioni e un tono che testimoniano il rispetto, si direbbe quasi sacro, per ciò che l'autore, chiunque esso sia, ha composto, facendo chiaramente intendere che chi acquista un testo musicale non è autorizzato a cambiarlo a proprio piacimento. Appare perciò evidente che il voler eventualmente assecondare la volontà del mecenate, "ingiuriando" il lavoro dell'autore, non gli impedisce di esprimere liberamente e con una significativa forza la propria visione riguardo al rispetto della volontà del compositore.

In conclusione, alcune lettere e i documenti relativi alle edizioni delle sue musiche offrono una prospettiva interessante per chiarire il concetto di proprietà intellettuale sviluppato dal piranese, pur in un contesto caratterizzato dalla scarsità o dall'assenza di strumenti in grado di tutelare la proprietà delle composizioni di un autore. Oltre ad una scontata attenzione per le proprie composizioni, Tartini dimostrò, con una certa coerenza e costanza nel tempo, un rispetto per la proprietà dell'opera altrui: sia che gli autori fossero allievi, sia che fossero compositori estranei alla sua cerchia, o addirittura, come nel caso di Geminiani e Corelli, già defunti. Il suo atteggiamento, o per dirla con le sue parole, il suo "sentimento", appare fortemente influenzato da un senso dell'onore, che come afferma Sergio Durante "was in fact an essential tool for any trustworthy trader", e anche dal suo personale carattere che lo portò ad enfatizzare il rispetto per determinate regole morali non scritte e raramente osservate..$^{32}$

${ }^{30}$ Fanzago, Orazione del signor abate, 47, nota b.

${ }^{31}$ US-BEm, Italian MS 209-210.

32 Durante, "Tartini and his Texts," 184. 


\section{Fonti}

Museo internazionale e biblioteca della musica di Bologna (I-Bc), Carteggio Martini: I.017.015: lettera di Giuseppe Tartini a padre Giovanni Battista Martini (3 dicembre 1739) I.023.083: lettera di Francesco Benzoni a padre Giovanni Battista Martini (14 novembre 1739)

I.017.017: lettera di Giuseppe Tartini a padre Giovanni Battista Martini (26 febbraio 1740)

\section{Bibliografia}

Barazzoni, Beatrice, "Vandini, Antonio." In Die Musik in Geschichte und Gegenwart, 2. ed., edited by Ludwig Finscher, Personenteil, 16:1312-1313. Kassel: Bärenreiter; Stuttgart: Metzler, 2006.

Bisi, Teresa Sofia. “Contributo ad un'edizione critica dei sei concerti opera prima - libro primo di Giuseppe Tartini.” Tesi di laurea, Università degli Studi di Padova, Facoltà di Lettere e Filosofia, 1995-1996.

Brainard, Paul. “Die Violinsonaten Giuseppe Tartinis.” Tesi di dottorato, Universität zu Göttingen, 1959.

____ . "Le Sonate a tre di Giuseppe Tartini: un sunto bibliografico." Rivista italiana di musicologia 4 (1969): 102-126.

___ L L L sonate per violino di Giuseppe Tartini: catalogo tematico. Padova: Accademia Tartiniana; Milano: Carisch, 1975.

Canale, Margherita. "Fonti per una ricostruzione della didattica di Tartini nella 'Scuola della Nazioni'.” Muzikološki zbornik 28 (1992): 15-24.

Canale Degrassi, Margherita. "I concerti solistici di Giuseppe Tartini. Testimoni, tradizione e catalogo tematico.” Tesi di dottorato, Università degli Studi di Padova, 2009-2010.

De Vecchis, Chiara e Traniello, Paolo. La proprietà del pensiero. Il diritto d'autore dal Settecento ad oggi. Roma: Carocci, 2012.

Dizionario Enciclopedico della Musica e dei Musicisti. Edited by Alberto Basso. Le Biografie, s.v. "Bini, Pasquale (Pasqualino)", 1: 533-534. Torino: Utet, 1985.

Duckles, Vincent e Elmer, Minnie, eds. Thematic Catalog of a Manuscript Collection of Eighteenth-Century Italian Instrumental Music, in the University of California, Berkeley, Music Library. Berkeley, Los Angeles: University of California Press, 1963.

Durante, Sergio. "Tartini and his Texts." In Studies on Mozart and the $18^{\text {th }}$ Century, a cura di Sergio Durante, 167-200. Lucca: LIM, 2007.

Fanzago, Francesco. Orazione del signor abate Francesco Fanzago padovano delle lodi di Giuseppe Tartini [...]. Padova: Conzatti, 1770.

Ferretti, Luca. "Giuseppe Tartini e le Marche: primi risultati di una ricerca.” In Tartini: il tempo e le opere, a cura di Maria Nevilla Massaro e Andrea Bombi, 37-65. Bologna: Il Mulino, 1994.

Frasson, Leonardo. "Giuseppe Tartini: primo violino e capo di concerto nella Basilica del Santo. L'uomo e l'artista.” Il Santo 12, no. 1-2 (1972): 65-152.

Guillo, Laurent. "Legal Aspects.” In Music Publishing in Europe : 1600-1900 : Concepts and 
Issues, Bibliography, edited by Rudolf Rasch, 115-138. Berlin: Berliner WissenschaftsVerlag, 2005.

Hansell, Sven e Massaro, Maria Nevilla. "Vandini, Antonio." In Grove Music Online, Oxford Music Online. Accesso il 30 dicembre 2013. (http://www.oxfordmusiconline.com.) Hortis, Attilio. "Lettere di Giuseppe Tartini trascritte dalle autografe dell'Archivio di Pirano." Archeografo Triestino, Nuova Serie 10 (1884): 209-229.

Hunter, David. "Music Copyright in Britain to 1800." Music \& Letters 67, no. 3 (1986): 269-282.

Lenneberg, Hans, ed. The Dissemination of Music: Studies in the History of Music Publishing. Lausanne: Gordon and Breach, c.1994.

Lozzi, Carlo. “Tartini e Bini.” La Cronaca Musicale 1, no. 6 (1896): 182-185.

Marconato, Alessandra, "Per un'edizione critica dei sei concerti dell'opera prima, libro secondo di Giuseppe Tartini.” Tesi di laurea, Università degli Studi di Padova, Facoltà di Lettere e Filosofia, 1998-1999.

Pasini, Ferdinando. “Il Tartini a Giuseppe Valeriano Vannetti.” Pagine Istriane 4, n. 1-2 (1906): 1-13.

Petrobelli, Pierluigi. "La scuola di Tartini in Germania e la sua influenza." In Pierluigi Petrobelli, Tartini, le sue idee e il suo tempo, 81-100. Lucca: LIM, 1992.

___ . "Tartini, Algarotti e la corte di Dresda." In Pierluigi Petrobelli, Tartini, le sue idee e il suo tempo, 51-64. Lucca: LIM, 1992.

____ "Una presenza di Tartini a Parma nel 1728." In Pierluigi Petrobelli, Tartini, le sue idee e il suo tempo, 65-79. Lucca: LIM, 1992.

Pohlmann, Hansjörg. Die Frühgeschichte des musikalischen Urheberrechts (ca. 1400-1800).

Neue Materialen zur Entwicklung des Urheberrechtsbewusstseins der Komponisten. Kassel: Bärenreiter, 1962.

Rabin, Ronald J. e Zohn, Steven. "Arne, Handel, Walsh, and Music as Intellectual Property: Two Eighteenth-Century Lawsuits.” Journal of the Royal Musical Association 120, no. 1 (1995): 112-145.

Radiciotti, Giuseppe. "Aggiunte e correzioni ai dizionari biografici dei musicisti." Sammelbände der Internationalen Musikgesellschaft 15, no. 4 (1914): 566-586.

Rasch, Rudolf, ed. Music Publishing in Europe: 1600-1900: Concepts and Issues, Bibliography. Berlin: Berliner Wissenschafts-Verlag, 2005.

____ "«Il cielo batavo». I compositori italiani e le edizioni olandesi delle loro opere strumentali nel primo Settecento." Analecta Musicologica 32, (2002): 237-266.

Rostirolla, Giancarlo. "L'editoria musicale a Roma nel Settecento.” In Le Muse Galanti: la musica a Roma nel Settecento, a cura di Bruno Cagli, 121-176. Roma: Istituto della Enciclopedia Italiana, 1985.

Ruggeri, Vanessa. “Contributo per un'edizione critica dei sei concerti opera seconda di Giuseppe Tartini." Tesi di laurea, Università degli Studi di Padova, Facoltà di Lettere e Filosofia, 2000-2001.

Sartori, Antonio. Archivio Sartori. Documenti di storia e arte francescana. Vol. III/1, Evoluzione del francescanesimo nelle Tre Venezie. Monasteri, contrade, località, abitanti di Padova medioevale, a cura di Giovanni Luisetto. Padova: Biblioteca Antoniana, Basilica del Santo, 1988. 
Small, John. "Johann Christian Bach Goes to Law." The Musical Times 126, no. 1711 (1985): 526- 529.

___ . "The Development of Musical Copyright." In The Music Trade in Georgian England, a cura di Michael Kassler, 233-386. Farnham, Surrey: Ashgate, 2011.

Vio, Gastone. "Note biografiche su Girolamo Ascanio Giustinian.” In Benedetto Marcello: la sua opera e il suo tempo, Atti del Convegno internazionale: Venezia, 15-17 dicembre 1986, a cura di Claudio Madricardo e Franco Rossi, 61-74. Firenze: L. S. Olschki, 1988.

Viverit, Guido. "Dissemination and Tradition of Tartini's Compositions within the 'School of the Nations'.” Ad Parnassum 11, no. 22 (2013): 87-98. 


\section{GIUSEPPE TARTINI IN INTELEKTUALNA LASTNINA GLASBE V 18. STOLETJU}

\section{Povzetek}

V 18. stoletju se je v Evropi, zlasti v Veliki Britaniji in Franciji, razvnela živahna debata o intelektualni lastnini avtorskih del, posebno na literarnem področju. Čeprav počasi, so se prav v tem obdobju razvile nekatere pomembne oblike zaščite, predhodnice pravice, ki je danes v kontinentalni Evropi znana kot avtorska pravica, v anglosaškem prostoru pa kot »copyright« (založniška pravica). Istočasno pa na področju glasbe ni bilo orodij, ki bi jih avtor ali založnik imela na voljo za zaščito lastnine nekega dela, ali pa niso bila učinkovita. Dokaz za to je cvetoči trg nepooblaščenih izdaj.

Giuseppe Tartini predstavlja zanimiv primer koncepta »intelektualne lastnine« glasbenih del v 18. stoletju. S podatki, ki se nanašajo na odnos med Tartinijem in izdajatelji njegove glasbe, ter po zaslugi dokazov, ki jih nudijo nekatera njegova pisma, je mogoče osvetliti različne sicer zanemarjene aspekte, kot so glasbeno avtorstvo, avtorjev nadzor nad svojim delom, spoštovanje do avtorjevih ustvarjalnih namenov in opredelitev ekonomske vrednosti avtorjevih skladb.

Tartinijeva glasba se je že za časa njegovega življenja širila tako v rokopisih kot tudi tiskanih oblikah, a le dva opusa sonat je tudi sam avtoriziral, op. 1, ki ga je izdal Michel Charles Le Cène (Amsterdam, 1734), in op. 2, ki je izšel v Rimu pri Antoniu Cletonu (1745). V pismu Giuseppu Valerianu Vannettiju Tartini natančno opisuje dogovore s svojimi izdajatelji.

A večji del Tartinijevih del je zaradi nepoštenja nekaterih z njim povezanih oseb (učenci in poklicni kopisti) krožil brez njegovega dovoljenja. Nekateri dokumenti pričajo o neuspešnih skladateljevih poskusih nadzora kopistov. Čeprav je bil Tartini jasno razočaran nad neavtoriziranim kroženjem svojih del, pa so prav ta njegovo ime ponesla po vsej Evropi.

Njegova pisma vsebujejo informacije o ideji »intelektualne lastnine«, kot jo je razumel sam, in ne samo v zvezi z lastnimi skladbami, temveč tudi deli drugih glasbenikov (vključno z njegovimi učenci). Razprava obravnava dva taka primera. Prvi se ukvarja s skladbami, ki jih je konec leta 1739 napisal Tartinijev učenec Pasqualino Bini, drugi pa obravnava Corellijeve sonate op. 5 (tiste, ki jih je Francesco Geminiani priredil v concerte grosse). Oba primera kažeta na Tartinijevo zavedanje o pomembnosti intelektualne lastnine del in njegovo globoko spoštovanje do drugih glasbenikov. 